\title{
Review of non-invasive urinary biomarkers in bladder cancer
}

\author{
Hyung-Ho Lee, Sung Han Kim \\ Department of Urology, Urological Cancer Center, Research Institute and Hospital of National Cancer Center, Goyang, Korea \\ Contributions: (I) Conception and design: SH Kim; (II) Administrative support: SH Kim; (III) Provision of study materials or patients: SH Kim; (IV) \\ Collection and assembly of data: All authors; (V) Data analysis and interpretation: None; (VI) Manuscript writing: All authors; (VII) Final approval of \\ manuscript: All authors. \\ Correspondence to: Sung Han Kim. Department of Urology, Urological Cancer Center, Research Institute and Hospital of National Cancer Center, \\ 323 Ilsan-ro, Ilsandong-gu, Goyang-si Gyeonggi-do 10408, Korea. Email: urojy@ncc.re.kr.
}

\begin{abstract}
Bladder cancer (BC) is the sixth-most prevalent cancer. The standard diagnostic tool of $\mathrm{BC}$ is cystoscopy, whereas cystoscopy has several disadvantages in terms of symptomatic invasiveness and operatordependency. The urinary markers are attractive because the testing is non-invasive and cost-efficient, and sample collection is easy. Urinary marker is thereby a good tool to detect exfoliated tumor cell in the urine samples for the diagnosis and therapeutic surveillance of BC to supplement the limitations of the cystoscopy. However, they are not recommended as a population-based screening tool because of the low rate of BC prevalence. Although both cystoscopy and urine cytology improve BC diagnostic power, the field still needs additional non-invasive, cost-effective, and highly sensitive and specific diagnostic tools. Various urinary markers with different mechanisms and different targets have been developed and under investigation in these days. However, the accuracy of the urinary marker including its sensitivity and specificity is the most important factor for the diagnosis and surveillance in cancer that this review deals with multiple FDAapproved and non-FDA approved commercialized urinary markers with their accuracy in different purposes for BC. We then discuss more about the potential candidate targets for the future urinary markers in BC
\end{abstract}

Keywords: Bladder cancer (BC); urine; marker; sensitivity; specificity

Submitted May 04, 2020. Accepted for publication Jun 18, 2020.

doi: $10.21037 /$ tcr-20-1990

View this article at: http://dx.doi.org/10.21037/tcr-20-1990

\section{Introduction}

Bladder cancer (BC) is the sixth-most prevalent cancer, with age-standardized incidence rates of 9.6 and 2.4 per 100,000 for men and women, respectively (1). The current gold standard for diagnosing BC is cystoscopic visualization of bladder tumors, which has an overall sensitivity of $62-84 \%$ and specificity of $43-98 \%$ (2). However, cystoscopy has a limited ability to diagnose small papillary BC and BC carcinoma in situ (CIS) and is cost-intensive, invasive, and operator-dependent.

The degradation of cells, DNA, and RNA in urine samples depends on time and temperature, resulting in a variable quantity and quality of cell and DNA molecules and leading to highly variable sensitivities and specificities, which causes overestimations of the performance characteristics of urinary biomarker tests (3-9). However, urinary biomarkers are attractive because the testing is noninvasive and cost-efficient, and sample collection is easy. Urine cytology, the most common urinary biomarker test used for BC diagnosis and screening, involves examining voided urine and detecting exfoliated urothelial BC cancer cells that enter the urine through contact with the urinary tract (10).

Many current urinary biomarker tests have various applications. For example, clinicians analyze urinary biomarkers before and after cystoscopy or transurethral resection surgery to detect recurring or progressing $\mathrm{BC}$ after intravesical instillation or systemic therapy (11). Additionally, tumor characteristics during surgery provide information about potential tumor aggressiveness and invasiveness. This information is used to decide upon the 
appropriate resection margin, surgical depth, and whether to include the detrusor muscle, especially for high-grade tumors and CIS $(12,13)$.

Postoperative surveillance of urinary biomarkers is a potential non-invasive replacement for cystoscopy after transurethral resection surgery (14). Urinary biomarkers can be used for therapeutic surveillance after either neoadjuvant chemotherapy, adjuvant chemotherapy, or intravesical instillation $(15,16)$. However, they are not recommended as a population-based screening tool because of the low rate of $\mathrm{BC}$ prevalence (11). Although both cystoscopy and urine cytology improve BC diagnostic power, the field still needs additional non-invasive, cost-effective, and highly sensitive and specific diagnostic tools. Here, we discuss and compare several potential urinary biomarkers.

\section{Urinary biomarkers}

\section{FDA-approved urinary biomarkers}

\section{Urine cytology}

Urine cytology is the most reliable test among urinary biomarker tools for detecting exfoliated tumor cells in urine, with an overall sensitivity and specificity of up to $48 \%$ and $86 \%$, respectively $(17,18)$. It complements both primary diagnosis and recurrence surveillance of high-grade or CIS BC treated with intravesical therapy, with $38-90 \%$ sensitivity and $98-100 \%$ specificity. However, its sensitivity for low-grade BC is only $4-31 \%$, and the $12 \%$ false-positive rate reflects a poor ability to screen out patients with inflammation, atypical urothelial cells, or a history of other cancer therapies (17-20).

Urine cytology also has limitations in terms of discrepancies between cancer types and inter-observer variability, especially for patients with recurrent inflammation or previous immunotherapy. In addition, the interpretation of cystoscopy results is more reproducible than that of urine cytology results; therefore, when atypical urothelial cells are detected using urine cytology, the cytology results should not stand alone as a diagnostic replacement for cystoscopy.

\section{Bladder Tumor Antigen (BTA) stat and BTA TRAK (Polymedco Inc., Cortlandt, NY, USA)}

The BTA stat is a commercially available protein biomarker test that uses an immunochromatographic assay to detect BTA in previously diagnosed BC patients for the purpose of surveillance (15). The BTA stat and BTA TRAK tests detect the human complement factor $\mathrm{H}$ and complement factor H-related proteins that are involved in cancer cell growth and evasion of the host immune system; these levels increase during the invasion of BC cells (21-23). The sensitivity and specificity of BTA stat are $57-83 \%$ and $60-$ $92 \%$, respectively, while those of BTA TRAK are $73-77 \%$ and $45-81 \%$, respectively $(6,24,25)$.

For surveillance purposes, the overall sensitivity of BTA stat is superior to that of urine cytology, but the specificity is inferior (26). BTA TRAK also has a sensitivity superior to that of urine cytology and a higher sensitivity for high-grade and low-grade (Ta and T1) BC. However, BTA tests cannot replace urine cytology because of their inferior specificity (27) and high false positive rate caused by detection of benign diseases such as hematuria, urinary tract infection, stones, and in situ ureteral stenting $(4,25,28-30)$.

\section{NMP22 kit and NMP22 BladderChek (Alere, Waltham, MA, USA)}

The NMP22 kit and NMP22 BladderChek are both ELISA tests that target the NMP22 protein, which is more prevalent in $\mathrm{BC}$ cells than in the normal urothelium released in urine during apoptosis of urothelial cells $(31,32)$. The overall sensitivity and specificity of the NMP22 kit, which is used for surveillance (33), are 52-59\% and $87-89 \%$, respectively (33-35). The sensitivity and specificity of NMP22 BladderChek, which is used for diagnostics, are $62-75 \%$ and $70-83 \%$, respectively (33). The limitation of these tests is the high false positive rate caused by detection of benign conditions $(29,36)$.

Compared with urine cytology, the NMP22 test has a higher diagnostic sensitivity for microscopic hematuria $(3,31)$ and better detection power for low-grade, lowstage, and high-grade $\mathrm{BC}$ due to the increasing rates of cell apoptosis present in these disease conditions (37). However, the NMP22 test cannot replace urine cytology because it has a lower specificity (38).

\section{ImmunoCyt/uCyt+ assay (Scimedx Inc., Denville, NJ, USA)}

The ImmunoCyt/uCyt+ assay is the only test available for therapeutic follow-up and applies a combination of urine cytology and immunohistochemical staining using fluorescent-labeled monoclonal antibodies. The assay detects exfoliated $\mathrm{BC}$ cells using the carcinoembryonic antigen and two BC-associated mucins (LDQ10 and M344). An overall specificity ranging from $62 \%$ to $84 \%$ and 
sensitivity ranging from $67 \%$ to $100 \%$ have been observed during use of the assay for diagnostic and surveillance purposes (28).

The high sensitivity of this assay for detecting increased pathological grades as well as low-grade, low-risk BC $(33,39-43)$ is sufficient to make cystoscopy unnecessary (14). Limitations include the necessity for special laboratory equipment and skillful, experienced assay interpreters (44) and a lower specificity than that of urine cytology. It also has a high false positive rate caused by detection of benign diseases and an especially decreased specificity ( $67 \%)$ for hematuria (45).

\section{UroVysion (Vysis, Abott Molecular Inc., Chicago, IL, USA)}

The UroVysion uses a multi-target fluorescence in situ hybridization assay to detect aneuploidy in chromosome copy numbers 3, 7, 17, and 9p21 and identify loss of the P16 tumor-suppressor gene (46). The test has a sensitivity (57.1$84 \%$ ) and specificity (78-92\%) superior to those of urine cytology, especially for low-grade BC $(14,47,48)$. This test is especially useful for detecting $\mathrm{BC}$ in high-risk patients with equivocal cystoscopic findings and atypical cytology $(14,49)$, confirming Bacillus Calmette-Guerin (BCG) responsiveness in non-responders (50-53), and detecting $\mathrm{BC}$ recurrence in non-muscle invasive bladder cancer (NMIBC) patients with negative cystoscopy but suspicious cytology results (54).

Limitations include the necessity for laboratory equipment and test reading experience as well as timeconsuming specimen processing, which results in high costs $(44,55)$. There is a high false positive rate, especially for patients tested within 12 months of a bladder biopsy. The presence of other primary tumors with chromosomal aberrations also contributes to the false positive rate (56).

\section{Summary}

Currently, cystoscopy is the standard BC diagnostic tool. None of the urinary biomarkers can replace cystoscopy because they fail to diagnose $18-43 \%$ of BCs and yield a $12-26 \%$ false positive rate. However, urinary biomarkers are the best tool for overcoming cystoscopy's limitations and enhancing diagnostic accuracy. Compared to the popular urine cytology, the BTA stat, ImmunoCyt, and Urovision tests present higher sensitivities when used as diagnosis and surveillance tools, although they all have lower specificities, especially for low-grade BC, than cytology.

\section{Non-FDA approved urinary biomarkers}

ADXBLADDER (Arquer Diagnostics LTD, Sutherlands, UK)

ADXBLADDER, a protein biomarker assay, uses ELISA to detect mini-chromosome maintenance protein 5 (MCM 5), which is present during DNA replication and overexpressed in hematuric BC patients $(8,57)$. The overall sensitivity and specificity are estimated to be $76 \%$ and $69 \%$, respectively, although the sensitivity increases up to $95-97 \%$ for highrisk and muscle-invasive $\mathrm{BC}$. The negative predictive value (NPV) is $97.1 \%$ overall and $99.7 \%$ for high-risk BC (58). Therefore, this biomarker might be either a potential replacement for or an adjunctive to urine cytology as a BC diagnostic tool. The advantage of this test is that its diagnostic accuracy is not influenced by any benign conditions.

\section{UBC $^{\circledR}$ Rapid test and UBC ELISA kit}

The $\mathrm{UBC}^{\circledast}$ Rapid test is a point-of-care ELISA assay that uses an immunochromatographic method to detect soluble fragments of cytokeratin 8 and 18 of the urinary BC antigen, which relate to tumor invasion. This test detects high-grade NMIBC during diagnostics and follow-up more readily than it detects low-grade tumors, with a sensitivity of $50-75 \%$ and specificity of $82-93.8 \%(11,55,59,60)$. It is standardized and calibrated and thus independent of user, batch, and study site, and has the potential to be a sensitive and specific urinary protein biomarker test for identifying patients with high-grade tumors difficult to detect with cystoscopy.

\section{Uromonitor (U-Monitor, Porto, Portugal) and uromonitor-V2}

Uromonitor, a DNA biomarker test, uses real-time PCR to detect TERTp and FGFR 3 alterations in exfoliated tumor cells. The Uromonitor-V2 test has 100\% sensitivity, $83.3 \%$ specificity, $66.7 \%$ positive predictive value (PPV), and $100 \%$ NPV for surveillance of NMIBC (59). With an overall sensitivity of $73.5 \%$ and specificity of $73.2 \%$, Uromonitor performs similarly to cystoscopy across all stages and grades during recurrence surveillance after transurethral bladder resection (61), serving as an alternative test for patients ineligible for cystoscopy. Uromonitor achieves $100 \%$ sensitivity and $88.6 \%$ specificity when combined with cystoscopy (the sensitivity of cystoscopy combined with 
cytology is $86.7 \%$ ) (61). The detection rate increases from $62.5 \%$ for recurrent low-grade BC to $75 \%$ for recurrent high-grade BC. The Uromonitor test is also independent of the presence of inflammation or benign diseases.

\section{UroSEEK}

UroSEEK, a DNA biomarker assay, is more sensitive than cytology in both surveillance $(71 \%$ vs. $25 \%)$ and diagnostics $(95 \%$ vs. $43 \%)$ and detects genetic alterations in TERT, FGFR3, PIK3CA, TP53, HRAS, KRAS, ERBB2, $C D K N 2 A, M E T, M L L$, and $V H L(62,63)$. For primary BC diagnosis, high-risk patients and patients with atypical urine cytology are suitable test candidates. For surveillance, a sensitivity of $74-96 \%$, specificity of $72-88 \%$, and an NPV of $53-99 \%$ were reported (63). The limitations of this test are the low sensitivity of the next-generation sequencing (NGS) technique used to detect mutations and the poor performance during follow-up tests of previously diagnosed BC patients and patients with upper urinary tract urothelial carcinoma (3).

\section{CxBladder assay (Pacific Edge, NZ)}

CxBladder, a laboratory-developed transcriptomic panel assay, measures five mRNAs (IGFBP5, HOXA13, MDK, CDK1, and CXCR2) associated with BC and one mRNA associated with nonmalignant conditions to reduce the false positive rate, with an overall sensitivity of $82 \%$ (64). The CxBladderTriage has the advantage of profiling individualized risk by collecting clinical factor data, with a $100 \%$ detection rate for high-grade BC with gross hematuria (65). It also has a higher sensitivity for low-grade BC than urine cytology (68\% for pTa), with a specificity of $85 \%$. A sensitivity of $93 \%$ extends to all other evaluative tests across all stages and grades [sensitivity for low-grade: $86 \%$, sensitivity for high-grade: $95 \%$, NPV: $97 \%$, false negative rate: $1.5 \%$ (64)]. Therefore, cystoscopy can be avoided during follow-up of NMIBC cases (66). In comparison to the currently approved FDA tests, the sensitivity and NPV of CxBladder Monitor (91\%, 96\%) was significantly higher than those of urine cytology $(22 \%$, $87 \%)$, the NMP22 BC test $(26 \%, 87 \%)$, and NMP22 BladderChek (11\%, 86\%) (49).

\section{The XPERT BC Monitor (Cephaid, USA)}

XPERT BC Monitor, a transcriptomic panel test for five mRNAs (ABL1, CRH, IGF2, UPK 1B, and ANXA 10), has an overall sensitivity of $73 \%$ (superior to that of urine cytology) and an insignificant differential overall specificity of $90-91 \%$, comparable to that of urine cytology (67). The advantages of this test are its superior sensitivity (84\% vs. $33 \%, \mathrm{P}<0.001)$ and NPV (93\% vs. $76 \%, \mathrm{P}<0.001)$ for lowgrade $(77 \%)$ and $\mathrm{p}$ Ta disease $(82 \%)$ compared to urine cytology (13\% for low grade, $21 \%$ for pTa) (67).

\section{EpiCheck}

EpiCheck, an epigenetic DNA methylation biomarker test, targets 15 altered DNA methylation biomarkers to identify NMIBC recurrence. Its probability algorithm, EpiScore, has a sensitivity of $68.2 \%$, specificity of $88.0 \%$, and an NPV of $95.1 \%$. EpiCheck has a higher sensitivity $(62.3-68.2 \%$ vs. $33.3 \%)$ but a lower specificity $(86.3-88 \%$ vs. $98.6 \%)$ than urine cytology (68). The advantages of this test are its high sensitivity for high-grade BC, with a $82.9 \%$ NPV (69), and that the presence of inflammation in the urinary tract has no influential effect $(68,69)$. The disadvantages are a high cost and technically challenging procedure $(68,69)$.

\section{BC UroMark}

BC UroMark, a next-generation sequencing assay, is an epigenetic DNA methylation biomarker test targeting bisulfite. It uses a $150 \mathrm{CpG}$ loci biomarker panel and has 98\% sensitivity, $97 \%$ specificity, and $97 \%$ NPV (70).

\section{AssureMDx (MDx Health, USA)}

The AssureMDX panel assay is a somatic DNA methylation biomarker test using next-generation PCR sequencing for OTX1, ONECUT2 and TWIST1. It measures the mutational load of the FGFR3, TERT, and HRAS genetic panel with $93-97 \%$ sensitivity, $83-86 \%$ specificity, and 99\% NPV $(9,71)$ for high-risk patients with hematuria. The sensitivities are $81 \%$ and $57 \%$ for low-grade BC diagnosis and surveillance, respectively, and $94 \%$ and $72 \%$ for highgrade, respectively. The specificity for low-grade is $59 \%$; that for high-grade is $55 \%$, for surveillance. (71). This test produces a potential $77 \%$ reduction in unnecessary diagnostic cystoscopies $(9,72)$.

\section{TaqMan $^{\circledR}$ Arrays}

This $12+2$ gene-set panel is based on a qRT-PCR assay for BC detection and has a sensitivity of $98 \%$ and specificity of $99 \%$. For predicting BC aggressiveness, this test has a sensitivity of $79 \%$ ( $v s .86 \%$ in the control) and specificity of $92 \%$ (vs. $80 \%$ in the control) $(73,74)$. With a sensitivity of $81.48 \%$ and specificity of $91.26 \%$, the gene signature composed of two genes (GS_D2) performs equally to or better than cytology (75). 


\section{Investigational potent targets for urinary biomarkers}

\section{Proteolytic region of cytokeratin-19 (CYFRA 21-1)}

The CYFRA 21-1 can be detected as a soluble molecule in serum and other body fluids and has been recognized as a non-specific tumor biomarker for several neoplastic diseases, including $\mathrm{BC}$, in terms of diagnosis, prognosis, followup, and prompt recognition of recurrence. The CYFRA 21-1 test uses the ELISA method and has a sensitivity and specificity of $70-90 \%$ and $73-86 \%$, respectively (76).

\section{BLCA-4}

BLCA-4, a member of the NMP transcription factor family, is observed during the early stages of BC. The sensitivity and specificity are high, at $93-96 \%$ and $97-$ $100 \%$, respectively, but its role as a diagnostic biomarker for BC still requires further validation (60). Its potential is further strengthened by the absence of high BLCA-4 levels in patients with various benign conditions. Nevertheless, novel methods not requiring urine precipitation analysis are necessary to include the BLCA-4 assay in clinical practice.

\section{Soluble FAS (sFAS)}

sFAS, an anti-apoptotic protein, protects cancer cells from anti-tumor activity. The urinary level of sFAS, measured using ELISA tests, is associated consistently with increasing tumor grade, stages $\geq \mathrm{T} 1$, high NMP22 levels, and positive urine cytology results (77). The sensitivity and specificity are $88.03 \%$ and $89.19 \%$, respectively. sFAS produces more positive results than urine cytology, particularly for lowgrade, early-stage disease cases.

\section{Hyaluronic acid (HA)}

HA is involved in cell adhesion and proliferation. During tumor metastasis, the hyaluronidase enzyme (HAase) catalyzes HA reactions to facilitate cellular proliferation and motility (78). The HA test for NMIBC has a sensitivity and specificity of $87-100 \%$ and $89-98 \%$, respectively (5).

\section{Telomeres}

Urinary telomerase has a protective role in cancer cell chromosomes and is associated with the recurrence of NMIBC. The combination of telomerase activity measurement and cytology produces an increased sensitivity of $60-87 \%$ and a specificity of around $65-90 \%$ (66). The $\mathrm{PPV}$ is $83.3 \%$ for superficial stages, $42.1 \%$ for invasive stages, $83.3 \%$ for grade 1 tumors, $66.7 \%$ for grade 2 tumors, and $40.0 \%$ for grade 3 tumors; thus, telomerase activity correlates with lower grades and stages of BC.

\section{Bladder tumor fibronectin (BTF)}

BTF is a chemiluminescent immunometric test used for NMIBC surveillance after transurethral resection surgery, with a $91 \%$ sensitivity, $88 \%$ specificity, $93 \%$ NPV, and $73 \%$ PPV (79). BTF is more sensitive to high-grade and highstage tumors, with a positivity rate of $93.8 \%$ for invasive tumors versus only $73.1 \%$ for superficial tumors. Similar to our findings, several studies $(12,15)$ have reported that BTF sensitivity is between $81.6 \%$ and $100 \%$ for infiltrating tumors and between $70 \%$ and $73.3 \%$ for noninvasive tumors, this test has higher sensitivity and specificity than FDA-approved tests.

\section{Cytokines and chemokines}

The GM-CSF, IFN-alpha, IL-2, IL-6, IL-8, IL-10, IL18 , survivin, TNK-alpha, and TRAIL have also been used as urinary targets for predicting the therapeutic response to intravesical BCG therapy $(48,60)$. PDL-1 and CTLA-4 are also effective immunotherapy targets. Tumor-associated macrophages (TAMs, also known as M2 macrophages) are recruited by chemokines such as interleukin (IL)-4 and IL13 and promote tumor growth. A high density of tumorinfiltrating TAMs is associated with poor outcomes in various types of cancer, including BC.

\section{IGF2 and MAGE-A3}

IGF2 is a glycoprotein receptor on the cell membrane that relates to the PI3K-AKT pathway in BC. Melanomaassociated antigen 3 (MAGE-A3) is found in $43 \%$ of BCs as well as in the testes and placenta (74,80-83). In combination, IGF2 and MAGE-A3 have $81 \%$ sensitivity, $91 \%$ specificity, $87 \% \mathrm{PPV}$, and $88 \%$ NPV (80).

\section{Topoisomerase-II alpha (TopoIIA)}

Expression of the TopoIIA mutation is significantly higher in hematuric patients and MIBC patients than in patients with NMIBC and is significantly associated with higher NMIBC recurrence and progression rates (84). Recent studies reported a sensitivity of $73.8 \%$, specificity of $68.3 \%$, PPV of $64.2 \%$, and NPV of $78.6 \%$ using urinary real-time PCR (85).

\section{Future urinary biomarkers}

Advances in sequencing technology have enabled researchers to catalogue the spectrum of somatic mutations 
associated with urothelial $\mathrm{BC}(3)$ and substantially change therapeutic guidelines for the selection of molecular target therapies (4). Based on BC genomic information and given the advancements in highly sensitive PCR-based technology, various small, obtainable amounts of DNA from urine samples have become ideal candidates for biomarkers that enable early BC detection, as they feature small, cancer-specific alterations (86). The assembly of multiple urinary genetic biomarkers enhances the power to detect recurrence and to evaluate the therapeutic response of each patient $(7,87)$.

Studies over the past decade have consolidated the major molecular taxonomies and demonstrated potentially treatable gene mutations and their fusion proteins. These include the FGFR3-TACC3 fusion protein $(16,17)$ in pathways such as the mitogen-activated protein kinase (MAPK) pathway, BRAF, MAP2K1, MAP2K2 and the phosphoinositide 3-kinase (PI3K) pathway (for example, PIK3CA) in $61 \%$ of tumors (13-15) and the receptor tyrosine kinase-RAS pathway (39\% of metastatic tumors), including FGFR3 (14\%) and ERBB3 (13\%), and in the $\mathrm{PI} 3 \mathrm{~K}-\mathrm{RAC} \alpha$ serine/threonine-protein kinase (AKT)mechanistic target of rapamycin (mTOR) pathway (38\%), including PIK3CA (16\%) and AKT3 (12\%) (16).

Potential DNA-methylated urine-based genetic biomarkers include the ANF154, VIM, TWIST, SFRP1, SALL3, RUNX3, RASSF1, RARB, POU4F2, PCDH17, p16INK4A, p14ARF, ONECUT2, NID2, HOXA9, GSTP1, GDF15, EOMES, DAPK, CDH1, CCNA1, BCL2, and $A P C$ genes (7). The ZNF154 and POU4F2 genes have the greatest sensitivity for diagnosing BC $(>80 \%)$ and the combined TWIST1, OTX1, and ONECUT2 genes have been used in a commercialized product, called the AssureMDx test, for hematuric patients (88). The most exact combined urine panel for BC detection is that of the $S O X 1$, TIP2, MYOID, HOXA9_1, HOXA9_2, VAMP8, CASP8, SPP1, IFNG, CAPG, HLADPA1, and RIPK3 genes, with $100 \%$ sensitivity and $100 \%$ specificity (89).

cfDNA, long-noncoding RNA (lncRNA), and miRNA are also detectable in urine and are valuable tools for risk stratification and initial disease diagnoses $(90,91)$. For example, levels of ctDNA from the FGFR 3 and PIK3CA genes can be detected in urine and correlate with prognoses for recurrence and overall survival after intravesical therapy or cystectomy $(90,91)$. In voided urine, good targets for BC detection (92) include miR-137, miR-124-2, miR-124-3, miR-126, miR-152, miR-148b-3p, miR-182, and miR-199a, miR-3187-3p, miR-15b-5b, miR-27a-3p, miR-30a-5p,
miR-324-5p, miR-4738-3p, FBJ murine osteosarcoma viral oncogene homolog B mRNA, Regulator of Calcineurin 1 mRNA (with $99.3 \%$ sensitivity and $98.9 \%$ specificity), and lncRNA miR-497-HG (with 78.3\% sensitivity) (93-95).

Extracellular vesicles (EVs) with multiple cellular components such as proteins, DNA, and mRNA can also be found in urine and have a low degradation rate, due to the similarity between their membrane and that of the origin cell and their ability to avoid immune system detection (96). An innovative methodological device improved the detection of $\mathrm{E} V s$. in urine, with a sensitivity of $81.3 \%$ and specificity of $90.0 \%$ for BC diagnosis (97).

\section{Limitations of urinary biomarkers}

There are several limitations to be aware of when assessing urinary biomarkers, including the time-dependent degradation during storage of collected urine samples, urinary content processing time, and specialized detection tools like sedimentation PCR methodology. The immediate storage of urine samples at temperatures between -20 and $-80{ }^{\circ} \mathrm{C}$ for four weeks has been recommended for maintenance of DNA material (98). Another strategy for preventing testing bias is co-sedimenting normal cells, crystals, and other substances during downstream PCR analyses (99). When using NGS to detect tumor DNA in leukocyte-rich urine, the inconsistency of the concentrations of cells and DNA should be taken into consideration despite the higher sensitivity of cfDNA, because the ratio of tumor DNA to wildtype DNA is higher than that of DNA to urine sediments. Various alternative approaches that enrich the tumor DNA yield have been developed, such as sizebased cell selection, in which a filter captures smaller-sized normal cells along with tumor cells $(100,101)$. Repeated urine sampling is another way to increase testing sensitivity. Pooled urine samples collected from low-grade BC patients after at least 24 h have a sensitivity of $100 \%$, compared to only $75 \%$ when a single sample is analyzed (102).

\section{Conclusions}

This review presents many FDA-approved biomarkers and potential biomarker candidates. However, due to limited prospective and large longitudinal clinical trials of new urinary biomarker tests, the current international guidelines still do not recommend testing for other urinary biomarkers prior to performing cystoscopy. However, newer approaches focusing on molecular, genomic, and transcriptomic 
aberrations have promising accuracies, and these biomarkers may provide additional molecular information to guide individualized surveillance and therapy strategies in the future. This review may be used to inform the scope of future clinical trials.

\section{Acknowledgments}

Funding: This article is financially supported by the National Cancer Center Fund (NCC 1810241-2).

\section{Footnote}

Provenance and Peer Review: This article was commissioned by the Guest Editors (Dr. Ja Hyeon Ku, Dr. Hyeong Dong Yuk, and Dr. Hyung Suk Kim) for the series "Urothelial Carcinoma" published in Translational Cancer Research. The article was sent for external peer review organized by the Guest Editors and the editorial office.

Conflicts of Interest: Both authors have completed the ICMJE uniform disclosure form (available at http:// dx.doi.org/10.21037/tcr-20-1990). The series "Urothelial Carcinoma" was commissioned by the editorial office without any funding or sponsorship. The authors have no other conflicts of interest to declare.

Ethical Statement: The authors are accountable for all aspects of the work in ensuring that questions related to the accuracy or integrity of any part of the work are appropriately investigated and resolved.

Open Access Statement: This is an Open Access article distributed in accordance with the Creative Commons Attribution-NonCommercial-NoDerivs 4.0 International License (CC BY-NC-ND 4.0), which permits the noncommercial replication and distribution of the article with the strict proviso that no changes or edits are made and the original work is properly cited (including links to both the formal publication through the relevant DOI and the license). See: https://creativecommons.org/licenses/by-nc-nd/4.0/.

\section{References}

1. Observatory, T.G.C. Bladder Inc., 2019. Available online: http://gco.iarc.fr/today/data/factsheets/cancers/30Bladder-fact-sheet.pdf

2. Jocham D, Stepp H, Waidelich R. Photodynamic diagnosis in urology: state-of-the-art. Eur Urol 2008;53:1138-48.

3. Batista R, Vinagre N, Meireles S, et al. Biomarkers for bladder cancer diagnosis and surveillance: a comprehensive review. Diagnostics (Basel) 2020;10:39.

4. Lokeshwar VB, Shroeder GL, Selzer MG, et al. Bladder tumor markers for monitoring recurrence and screening comparison of hyaluronic acid-hyaluronidase and BTAStat tests. Cancer 2002;95:61-72.

5. Lokeshwar VB, Habuchi T, Grossman HB, et al. Bladder tumor markers beyond cytology: International Consensus Panel on bladder tumor markers. Urology 2005;66:35-63.

6. Tabayoyong W, Kamat AM. Current use and promise of urinary markers for urothelial cancer. Curr Urol Rep 2018;19:96.

7. Larsen LK, Lind GE, Guldberg P, et al. DNAmethylation-based detection of urological cancer in urine: overview of biomarkers and considerations on biomarker design, source of DNA, and detection technologies. Int J Mol Sci 2019;20:11.

8. Stoeber K, Halsall I, Freeman A, et al. Immunoassay for urothelial cancers that detects DNA replication protein Mcm5 in urine. Lancet 1999;354:1524-5.

9. van Kessel KEM, Beukers W, Lurkin I, et al. Validation of a DNA methylation-mutation urine assay to select Patients with hematuria for cystoscopy. J Urol 2017;197:590-5.

10. Sapre N, Anderson PD, Costello AJ, et al. Gene-based urinary biomarkers for bladder cancer: an unfulfilled promise? Urol Oncol 2014;32:48.e9-17.

11. Maas M, Bedke J, Stenzi A, et al. Can urinary biomarkers replace cystoscopy? World J Urol 2019;37:1741-9.

12. Babjuk M, Boehle A, Burger M, et al. EAU Guidelines on non-muscle-invasive urothelial carcinoma of the bladder: update 2016. Eur Urol 2017;71:447-61.

13. van der Aa MNM, Steyerberg EW, Bangma C, et al. Cystoscopy revisited as the gold standard for detecting bladder cancer recurrence: diagnostic review bias in the randomized, prospective CEFUB trial. J Urol 2010;183:76-80.

14. Schmitz-Dräger C, Bonberg N, Pesch B, et al. Replacing cystoscopy by urine markers in the follow-up of patients with low-risk non-muscle-invasive bladder cancer? An International Bladder Cancer Network project. Urol Oncol 2016;34:452-9.

15. Kamat AM, Dickstein RJ, Messetti F, et al. Use of fluorescence in situ hybridization to predict response to bacillus Calmette-Guerin therapy for bladder cancer: results of a prospective trial. J Urol 2012;187:862-7.

16. Alfred Witjes J, Lebret T, Comperat EM, et al. Updated 
2016 EAU guidelines on muscle-invasive and metastatic bladder cancer. Eur Urol 2017;71:462-75.

17. Leiblich A. Recent developments in the search for urinary biomarkers in bladder cancer. Curr Urol Rep 2017;18:100.

18. Yafi FA, Brimo F, Steinberg J, et al. Prospective analysis of sensitivity and specificity of urine cytology and other urinary biomarkers for bladder cancer. Urol Oncol 2015;33:66.e25-31.

19. Lotan Y, Roehrborn CG. Sensitivity and specificity of commonly available bladder tumor markers versus cytology: results of a comprehensive literature review and meta-analyses. Urology 2003;61:109-18.

20. Burchardt M, Burchardt T, Shabsigh A, et al. Current concepts in biomarker technology for bladder cancers. Clin Chem 2000;46:595-605.

21. Sarosdy MF, Hudson MA, Ellis WJ, et al. Improved detection of recurrent bladder cancer using the Bard BTA stat test. Urology 1997;50:349-53.

22. Kinders R, Jones T, Root R, et al. Complement factor $\mathrm{H}$ or a related protein is a marker for transitional cell cancer of the bladder. Clin Cancer Res 1998;4:2511-20.

23. Malkowicz SB. The application of human complement factor H-related protein (BTA TRAK) in monitoring patients with bladder cancer. Urol Clin North Am 2000;27:63-73.

24. Glas AS, Roos D, Deutekom M, et al. Tumor markers in the diagnosis of primary bladder cancer. A systematic review. J Urol 2003;169:1975-82.

25. Duquesne I, Weisbach L, Aziz A, et al. The contemporary role and impact of urine-based biomarkers in bladder cancer. Transl Androl Urol 2017;6:1031-42.

26. Gutierrez Banos JL, del Henar Rebollo Rodrigo M, Antolin Juarez FM, et al. Usefulness of the BTA STAT test for the diagnosis of bladder cancer. Urology. 2001;57:685-9.

27. Thomas L, Leyh H, Marberger M, et al. Multicenter trial of the quantitative BTA TRAK assay in the detection of bladder cancer. Clin Chem 1999;45:472-7.

28. Lokeshwar VB, Soloway MS. Current bladder tumor tests: does their projected utility fulfill clinical necessity? J Urol 2001;165:1067-77.

29. Sharma S, Zippe CD, Pandrangi L, et al. Exclusion criteria enhance the specificity and positive predictive value of NMP22 and BTA stat. J Urol 1999;162:53-7.

30. Nasuti JF, Gomella LG, Ismail M, et al. Utility of the BTA stat test kit for bladder cancer screening. Diagn Cytopathol 1999;21:27-9.

31. Moonen PMJ, Kiemeney LALM, Witjes JA. Urinary
NMP22 BladderChek test in the diagnosis of superficial bladder cancer. Eur Urol 2005;48:951-6.

32. Landman J, Chang Y, Kavaler E, et al. Sensitivity and specificity of NMP-22, telomerase, and BTA in the detection of human bladder cancer. Urology 1998;52:398-402.

33. Chou R, Gore JL, Buckley D, et al. Urinary biomarkers for diagnosis of bladder cancer: a systematic review and meta-analysis. Ann Intern Med 2015;163:922-31.

34. Schiess R, Wollscheid B, Aebersold R. Targeted proteomic strategy for clinical biomarker discovery. Mol Oncol 2009;3:33-44.

35. Chakraborty A, Dasari S, Long W, et al. Urine protein biomarkers for the detection, surveillance, and treatment response prediction of bladder cancer. Am J Cancer Res 2019;9:1104-17.

36. Shariat SF, Marberger MJ, Lotan Y, et al. Variability in the performance of nuclear matrix protein 22 for the detection of bladder cancer. J Urol 2006;176:919-26.

37. Todenhöfer T, Hennenlotter J, Aufderklamm S, et al. Individual risk assessment in bladder cancer patients based on a multi-marker panel. J Cancer Res Clin Oncol 2013;139:49-56.

38. Do an C, Pelit ES, Yildirim A, et al. The value of the NMP22 test for superficial bladder cancer diagnosis and follow-up. Turk J Urol 2013;39:137-42.

39. Comploj E, Mian C, Ambrosini-Spaltro A, et al. uCyt+/ ImmunoCyt and cytology in the detection of urothelial carcinoma: an update on 7422 analyses. Cancer Cytopathol 2013;121:392-97.

40. Mian C, Pycha A, Wiener H, et al. Immunocyt: a new tool for detecting transitional cell cancer of the urinary tract. J Urol 1999;161:1486-9.

41. Vriesema JL, Atsma F, Kiemeney LA, et al. Diagnostic efficacy of the ImmunoCyt test to detect superficial bladder cancer recurrence. Urology 2001;58:367-71.

42. Pfister C, Chautard D, Devonec M, et al. Immunocyt test improves the diagnostic accuracy of urine cytology: results of a French multicenter study. J Urol 2003;169:921-4.

43. Mian C, Maier K, Comploj E, et al. uCyt+/ImmunoCyt in the detection of recurrent urothelial carcinoma: an update on 1991 analyses. Cancer 2006;108:60-5.

44. Xylinas E, Kluth LA, Rieken M, et al. Urine markers for detection and surveillance of bladder cancer. Urol Oncol 2014;32:222-9.

45. Olsson H, Zackrisson B. ImmunoCyt a useful method in the follow-up protocol for patients with urinary bladder carcinoma. Scand J Urol Nephrol 2001;35:280-2. 
46. Sokolova IA, Halling KC, Jenkins RB, et al. The development of a multitarget, multicolor fluorescence in situ hybridization assay for the detection of urothelial carcinoma in urine. J Mol Diagn 2000;2:116-23.

47. Mowatt G, Zhu S, Kilonzo M, et al. Systematic review of the clinical effectiveness and cost-effectiveness of photodynamic diagnosis and urine biomarkers (FISH, ImmunoCyt, NMP22) and cytology for the detection and follow-up of bladder cancer. Health Technol Assess 2010;14:1-331, iii-iv.

48. Shariat SF, Canto EI, Kattan MW, et al. Beyond prostatespecific antigen: new serologic biomarkers for improved diagnosis and management of prostate cancer. Rev Urol 2004;6:58-72.

49. Lotan Y, O'Sullivan P, Raman JD, et al. Clinical comparison of noninvasive urine tests for ruling out recurrent urothelial carcinoma. Urol Oncol 2017;35:531. e15-e22.

50. Kipp BR, Karnes RJ, Brankley SM, et al. Monitoring intravesical therapy for superficial bladder cancer using fluorescence in situ hybridization. J Urol 2005;173:401-4.

51. Savic S, Zlobec I, Thalmann GN, et al. The prognostic value of cytology and fluorescence in situ hybridization in the follow-up of nonmuscle-invasive bladder cancer after intravesical Bacillus Calmette-Guerin therapy. Int J Cancer 2009;124:2899-904.

52. Lodde M, Mian C, Mayr R, et al. Recurrence and progression in patients with non-muscle invasive bladder cancer: prognostic models including multicolor fluorescence in situ hybridization molecular grading. Int $\mathrm{J}$ Urol 2014;21:974.

53. Chang SS, Boorijan SA, Chou R, et al. Diagnosis and treatment of non-muscle invasive bladder cancer: aUA/ SUO guideline. J Urol 2016;196:1021-9.

54. Kim PH, Sukhu R, Cordon BH, et al. Reflex fluorescence in situ hybridization assay for suspicious urinary cytology in patients with bladder cancer with negative surveillance cystoscopy. BJU Int 2014;114:354-9.

55. Schmitz-Dräger BJ, Droller M, Lokeshwar VB, et al. Molecular markers for bladder cancer screening, early diagnosis, and surveillance: the WHO/ICUD consensus. Urol Int 2015;94:1-24.

56. Hajdinjak T. UroVysion FISH test for detecting urothelial cancers: meta-analysis of diagnostic accuracy and comparison with urine cytology testing. Urol Oncol 2008;26:646-51.

57. Mukhtar S, Perry MJA. Future prospects for bladder cancer biomarkers. BJU Int 2011;108:1541-3.
58. Dudderidge T, Stockley J, Nabi G, et al. A Novel, noninvasive Test Enabling Bladder Cancer Detection in Urine Sediment of Patients Presenting with Haematuria-A Prospective Multicentre Performance Evaluation of ADXBLADDER. Eur Urol Oncol 2020;3:42-6.

59. Lotan Y, Elias K, Svatek RS, et al. Bladder cancer screening in a high risk asymptomatic population using a point of care urine based protein tumor marker. J Urol 2009;182:52-7.

60. Urquidi V, Kim J, Chang M, et al. CCL18 in a multiplex urine-based assay for the detection of bladder cancer. PLoS One 2012;7:e37797.

61. Batista R, Vinagre J, Prazeres H, et al. Validation of a novel, sensitive, and specific urine-based test for recurrence surveillance of patients with non-muscle-invasive bladder cancer in a comprehensive multicenter study. Front Genet 2019;10:1237.

62. Springer SU, Chen CH, Rodriguez Pena MDC, et al. Non-invasive detection of urothelial cancer through the analysis of driver gene mutations and aneuploidy. Elife 2018;7:e32143.

63. Rodriguez Pena MDC, Springer SU, Taheri D, et al. Performance of novel non-invasive urine assay UroSEEK in cohorts of equivocal urine cytology. Virchows Arch 2020;476:423-9.

64. Kavalieris L, O'Sullivan P, Frampton C, et al. Performance characteristics of a multigene urine biomarker test for monitoring for recurrent urothelial carcinoma in a multicenter study. J Urol 2017;197:1419-26.

65. Kavalieris L, O’Sullivan PJ, Suttie JM, et al. A segregation index combining phenotypic (clinical characteristics) and genotypic (gene expression) biomarkers from a urine sample to triage out patients presenting with hematuria who have a low probability of urothelial carcinoma. BMC Urol 2015;15:23.

66. Brems-Eskildsen AS, Zieger K, Tolbod H, et al. Prediction and diagnosis of bladder cancer recurrence based on urinary content of hTERT, SENP1, PPP1CA, and MCM5 transcripts. BMC Cancer 2010;10:646.

67. Pichler R, Fritz J, Tulchiner G, et al. Increased accuracy of a novel mRNA-based urine test for bladder cancer surveillance. BJU Int 2018;121:29-37.

68. Witjes JA, Morote J, Cornel EB, et al. Performance of the bladder EpiCheck methylation test for patients under surveillance for non-muscle-invasive bladder cancer: results of a multicenter, prospective, blinded clinical trial. Eur Urol Oncol 2018;1:307-13.

69. Trenti E, D'Elia C, Mian C, et al. Diagnostic predictive 
value of the Bladder EpiCheck test in the follow-up of patients with non-muscle-invasive bladder cancer. Cancer Cytopathol 2019;127:465-9.

70. Feber A, Dhami P, Dong L, et al. UroMark-a urinary biomarker assay for the detection of bladder cancer. Clin Epigenetics 2017;9:8.

71. Beukers W, van der Keur KA, Kandimalla R, et al. FGFR3, TERT and OTX1 as a urinary biomarker combination for surveillance of patients with bladder cancer in a large prospective multicenter study. J Urol 2017;197:1410-8.

72. van Kessel KEM, Van Neste L, Lurkin I, et al. Evaluation of an epigenetic profile for the detection of bladder cancer in patients with hematuria. J Urol 2016;195:601-7.

73. Mengual L, Burset M, Ribal MJ, et al. Gene expression signature in urine for diagnosing and assessing aggressiveness of bladder urothelial carcinoma. Clin Cancer Res 2010;16:2624-33.

74. Mengual L, Ribal MJ, Lozano JJ, et al. Validation study of a noninvasive urine test for diagnosis and prognosis assessment of bladder cancer: evidence for improved models. J Urol 2014;191:261-9.

75. Ribal MJ, Mengual L, Lozano JJ, et al. Gene expression test for the non-invasive diagnosis of bladder cancer: a prospective, blinded, international and multicenter validation study. Eur J Cancer 2016;54:131-8.

76. Huang YL, Chen J, Yan W, et al. Diagnostic accuracy of cytokeratin-19 fragment (CYFRA 21-1) for bladder cancer: a systematic review and meta-analysis. Tumour Biol 2015;36:3137-45.

77. Svatek RS, Herman MP, Lotan Y, et al. Soluble Fas-a promising novel urinary marker for the detection of recurrent superficial bladder cancer. Cancer 2006;106:1701-7.

78. Sanchez-Carbayo M, Urrutia M, Romani R, et al. Serial urinary IL-2, IL-6, IL-8, TNFalpha, UBC, CYFRA 21-1 and NMP22 during follow-up of patients with bladder cancer receiving intravesical BCG. Anticancer Res 2001;21:3041-7.

79. Sagnak L, Ersoy H, Ozok U, et al. Predictive value of urinary interleukin-8 cutoff point for recurrences after transurethral resection plus induction bacillus CalmetteGuerin treatment in non-muscle-invasive bladder tumors. Clin Genitourin Cancer 2009;7:E16-23.

80. Yang SS, Calarco PG, Wivej NA. Biochemical properties and replication of murine intracisternal A particles during early embryogenesis. Eur J Cancer 1975;11:131-8.

81. Knowles MA, Platt FM, Ross RL, et al.

Phosphatidylinositol 3-kinase (PI3K) pathway activation in bladder cancer. Cancer Metastasis Rev 2009;28:305-16.

82. Dyrskjøt L, Zieger K, Lildal TK, et al. Expression of MAGE-A3, NY-ESO-1, LAGE-1 and PRAME in urothelial carcinoma. Br J Cancer 2012;107:116-22.

83. De Plaen E, Traversari C, Gaforio JJ, et al. Structure, chromosomal localization, and expression of 12 genes of the MAGE family. Immunogenetics 1994;40:360-9.

84. Kim EJ, Lee YS, Kim YJ, et al. Clinical implications and prognostic values of topoisomerase-II alpha expression in primary non-muscle-invasive bladder cancer. Urology 2010;75:1516.e9-e13.

85. Kim YH, Yan C, Lee IS, et al. Value of urinary topoisomerase-IIA cell-free DNA for diagnosis of bladder cancer. Investig Clin Urol 2016;57:106-12.

86. Heitzer E, Haque IS, Roberts CES, et al. Current and future perspectives of liquid biopsies in genomics-driven oncology. Nat Rev Genet 2019;20:71-88.

87. Trevethan R. Sensitivity, specificity, and predictive values: foundations, pliabilities, and pitfalls in research and practice. Front Public Health 2017;5:307.

88. Koch A, Joosten SC, Feng Z, et al. Analysis of DNA methylation in cancer: location revisited. Nat Rev Clin Oncol 2018;15:459-66.

89. Wang Y, Yu Y, Ye R, et al. An epigenetic biomarker combination of PCDH17 and POU4F2 detects bladder cancer accurately by methylation analyses of urine sediment DNA in Han Chinese. Oncotarget 2016;7:2754-64.

90. Todenhöfer T, Struss WJ, Seiler R, et al. Liquid biopsyanalysis of circulating tumor DNA (ctDNA) in bladder cancer. Bladder Cancer 2018;4:19-29.

91. Birkenkamp-Demtröder K, Christensen E, Nordentoft I, et al. Monitoring treatment response and metastatic relapse in advanced bladder cancer by liquid biopsy analysis. Eur Urol 2018;73:535-40.

92. Shindo T, Shimizu T, Nojima M, et al. Evaluation of urinary DNA methylation as a marker for recurrent bladder cancer: a 2-center prospective study. Urology 2018;113:71-8.

93. Eissa S, Safwat M, Matboli M, et al. Measurement of urinary level of a specific competing endogenous RNA network (FOS and RCAN mRNA/ miR-324-5p, miR4738-3p, /lncRNA miR-497-HG) enables diagnosis of bladder cancer. Urol Oncol 2019;37:292.e19-e27.

94. Hanke M, Hoefig K, Merz H, et al. A robust methodology to study urine microRNA as tumor marker: microRNA-126 and microRNA-182 are related to urinary bladder cancer. Urol Oncol 2010;28:655-61. 
95. Jiang X, Du L, Wang L, et al. Serum microRNA expression signatures identified from genome-wide microRNA profiling serve as novel noninvasive biomarkers for diagnosis and recurrence of bladder cancer. Int J Cancer 2015;136:854-62.

96. Liu YR, Ortiz-Bonilla CJ, Lee YF. Extracellular Vesicles in Bladder Cancer: Biomarkers and Beyond. Int J Mol Sci 2018;19:2822.

97. Liang LG, Kong MQ, Zhou S, et al. An integrated doublefiltration microfluidic device for isolation, enrichment and quantification of urinary extracellular vesicles for detection of bladder cancer. Sci Rep 2017;7:46224.

98. Bosschieter J, Bach S, Bijnsdorp IV, et al. A protocol for urine collection and storage prior to DNA methylation

Cite this article as: Lee HH, Kim SH. Review of non-invasive urinary biomarkers in bladder cancer. Transl Cancer Res 2020;9(10):6554-6564. doi: 10.21037/tcr-20-1990 analysis. PLoS One 2018;13:e0200906.

99. Lin SY, Linehan JA, Wilson TG, et al. Emerging utility of urinary cell-free nucleic acid biomarkers for prostate, bladder, and renal Cancers. Eur Urol Focus 2017;3:265-72.

100.Stasik S, Salomo K, Heberling U, et al. Evaluation of TERT promoter mutations in urinary cell-free DNA and sediment DNA for detection of bladder cancer. Clin Biochem 2019;64:60-3.

101.Andersson E, Steven K, Guldberg P. Size-based enrichment of exfoliated tumor cells in urine increases the sensitivity for DNA-based detection of bladder cancer. PLoS One 2014;9:e94023.

102.Dahl C, Guldberg P. DNA methylation analysis techniques. Biogerontology 2003;4:233-50. 